\title{
Chromosomal differentiation in Podisma pedestris: a third race
}

\author{
J. Gosálvez, \\ J. L. Bella and \\ G. M. Hewitt*
}

\author{
Departamento de Genética C-XV, Facultad de \\ Ciencias, Universidad Autónoma de Madrid, Madrid \\ 28049, Spain. \\ * School of Biological Sciences, University of East \\ Anglia, Norwich NR4 7TJ, England.
}

A new chromosomal race of the wingless grasshopper Podisma pedestris has been found in the Pyrenees in addition to two previously described in the Alps (XO and XY races). The differences which characterize the populations in the Pyrenees concern the amount, distribution and richness, in terms of base pair repeats, of the heterochromatic regions and the position of the nucleolus organizer regions in the $\mathrm{X}$ chromosome. While populations in the Alps have GC-rich procentric and distal heterochromatin, populations in the Pyrenees have more procentric heterochromatin formed by AT- plus GC-rich DNA in some chromosomes and absence of distal heterochromatin. Active NORs in the $X$ chromosome from alpine populations are located in the distal region, irrespective of the chromosomal race, while the active NOR in the $\mathrm{X}$ in the Pyrenean populations is proximally placed.

Hybrids produced from laboratory crosses between all the races are viable and show the expected heterozygous karyotypes but no sign of meiotic dysfunction, at least in the male.

\section{INTRODUCTION}

Podisma pedestris is an acridoid grasshopper, with its main area of distribution in Siberia. The European distribution is restricted to the Alps, Apennines and Pyrenees, where the populations of this wingless grasshopper occupy high mountain habitats. Most of the work on this grasshopper concerns two Alpine parapatric chromosomal races which meet in a narrow zone and form hybrids (Hewitt, 1975; Barton and Hewitt, 1981a; Hewitt and Barton, 1981; Halliday et al., 1983, 1984). These races have different sex-chromosome systems. One of them has the standard karyotype of the acridoids, $2 n=22+\mathrm{XO} / \mathrm{XX}$, while the other, which occupies an extensive area in the Alpes Maritimes, has a neo-XY sex-chromosome system, produced by a centric fusion between the original X, and a large autosome (John and Hewitt, 1970; Hewitt and John, 1972; Hewitt, 1975). Barton and Hewitt (1981b) and Nichols (1985) have shown partial inviability of hybrid individuals which helps to maintain this hybrid zone. Westerman and Hewitt (1985) have characterised both $\mathrm{XX} / \mathrm{XO}$ and Neo-XY chromosome races by using
C- and G-banding, as well as different fluorescent techniques, revealing that $\mathrm{C}$-heterochromatin in both races is relatively rich in Guanine-Cytosine (G-C) base pairs.

The populations of $P$. pedestris from the Pyrenees represent the westernmost extent of its European distribution. Since isolation between populations in the Alps and Pyrenees occurred during the last glacial retreat (some 13,000 years B.P.) both systems have been evolving in the absence of gene flow. Under these conditions one might expect that they have followed different pathways of evolution and this may be reflected in the structure of the genome and in chromosomal morphology. The aim of this paper is to investigate if this kind of divergence has occurred between the Alpine and Pyrenean populations.

This investigation has additional interest since populations of Podisma localized in southern regions of the Iberian Peninsula are described as a different species (Podisma ignatii) (MoralesAgacino, 1950), this could be due to a major differentiation in some populations isolated from those in the Pyrenees. 


\section{MATERIAL AND METHODS}

This study has been carried out using adults and embryos of Podisma pedestris. Young adult specimens from the Alps and the Pyrenees were collected in the field. Testes from males were fixed directly in 1:3 glacial acetic acid/ethanol, while gastric caeca and ovarioles obtained from females were removed and fixed after an eight hour abdominal injection with 0.05 per cent of colchicine in insect saline solution. Fourth instar insects were also collected from the Pyrenees and the Alps and the embryos obtained from laboratory crosses were treated with colchicine-saline solution for 15 minutes and fixed in 1:3 glacial acetic acid/ethanol.

Air dried chromosome preparations were made by squashing tissues in 45 per cent acetic acid, freezing them in liquid nitrogen and removing the coverslip with a razor blade. The acridine orange (AO)/Giemsa C-banding technique has been described previously (Bella et al., 1986). Fluorescence techniques were developed following Schweizer's procedures with minor modifications (Schweizer, 1976a, b; 1980, 1981 and personal communication). For DAPI counterstaining, slides were exposed to $1 \mu \mathrm{g} / \mathrm{ml}$ DAPI (Serva) in McIlvane Buffer $(p \mathrm{H} \mathrm{7.0)}$ for 30 minutes at room temperature in complete darkness. After washing in the same buffer, air dried slides were treated with $1 \mu \mathrm{g} / \mathrm{ml}$ Distamycin A (DA) (Serva) or $20 \mu \mathrm{g} / \mathrm{ml}$ Actinomycin D (AD) (Serva) in this buffer for 20 minutes, in the same conditions of darkness and temperature. After washing and air drying, they were mounted in a $1: 1$ mixture of Glycerol (Merck) and McIlvane Buffer.

For DA-Chromomycin $\mathrm{A}_{3}$ technique (DACMA), 2-3 day old slides were incubated in $0.5 \mathrm{mg} / \mathrm{ml} \mathrm{CMA} \mathrm{in} \mathrm{McIlvane} \mathrm{Buffer} \mathrm{for} 1$ hour, rinsed in deionized water and air dried. Conditions of temperature and darkness were the same as those explained for DAPI. They were then treated with DA, in the same way as for AD/DA-DAPI counterstaining, and finally mounted in $1: 1 \mathrm{Gly}$ cerol/McIlvane's containing $2.5 \mathrm{mM} \quad \mathrm{MgCl}_{2}$. They were incubated for three days at $37^{\circ} \mathrm{C}$. The silver staining used is that previously reported by Gosálvez et al. (1986).

Observations were carried out with a Zeiss Photo III microscope equipped with a $50 \mathrm{~W}$ epilluminator, neofluar objectives and the appropriate exciter and barrier filters. The photographs in the bright field microscope were made with Kodak AHU25 film and those in fluorescence with Valca F22 film.

\section{RESULTS}

\section{The Alpine populations}

The results obtained from these populations are similar to those reported by Westerman and Hewitt (loc. cit.). However, since new banding techniques have been used in this study, detailed results from $P$. pedestris in the Alps will be given.

Two different sex-chromosome races occur in the south-western Alpine populations of $P$. pedestris. The ancestral form has the standard acridoid complement $2 n=22+\mathrm{XO} / \mathrm{XX}$, where three pairs are considered as large $\left(\mathrm{L}_{1}-\mathrm{L}_{3}\right)$, six as medium $\left(\mathrm{M}_{4}-\mathrm{M}_{9}\right)$ and two pairs as small sized chromosomes $\left(S_{10}-S_{11}\right)$. The Neo-XY race has the characteristic sex chromosome system $2 n=20+$ neoXneoY/neoX/neoX. This neo-X chromosome has probably originated from a centromeric fusion between an $\mathrm{X}$ chromosome and the $L_{3}$ autosome (John and Hewitt, 1970; Westerman and Hewitt, 1985).

Slides treated to produce C-bands but stained with $\mathrm{AO}$, show the existence of paracentromeric C-heterochromatin in all the members of the complement in both $\mathrm{XO}$ and Neo-XY races, as well as regions of distal heterochromatin on all the medium sized chromosomes (fig. 1(a); Fig. 1(c)). The megameric $\mathrm{M}_{7}$ pair shows interstitial C-positive bands. The $\mathrm{X}$ chromosome in the alpine populations can be easily recognized with this technique because it is the longest of the medium sized pairs, showing both paracentromeric and distal heterochromatin, and the intense green fluorescence of the distal band contrasts with the orange obtained in the autosomes (fig. 1(a)). It should be noted that the terminal C-heterochromatin on the neo-X chromosome shows the same intense green fluorescence as that on the single X (Fig. 1(c)).

The use of C-banding and post-staining with either AO or Giemsa reveals the same chromosome bands, except for the presence of a thin proximal AO-fluorescent band, probably on the $L_{2}$ autosome, which is not visible after Giemsa C-banding (fig. 1(b)).

DA-CMA counterstaining shows intense yellow fluorescence of all the C-positive heterochromatin in both races. The absence of a positive response to AD-DAPI in the present work agrees completely with the absence of AT-rich DNA reported by Westerman and Hewitt (1985) using Hoechst 33258.

\section{The Pyrenean populations}

All the individuals examined from Pyrenean populations have the basic chromosome complement 

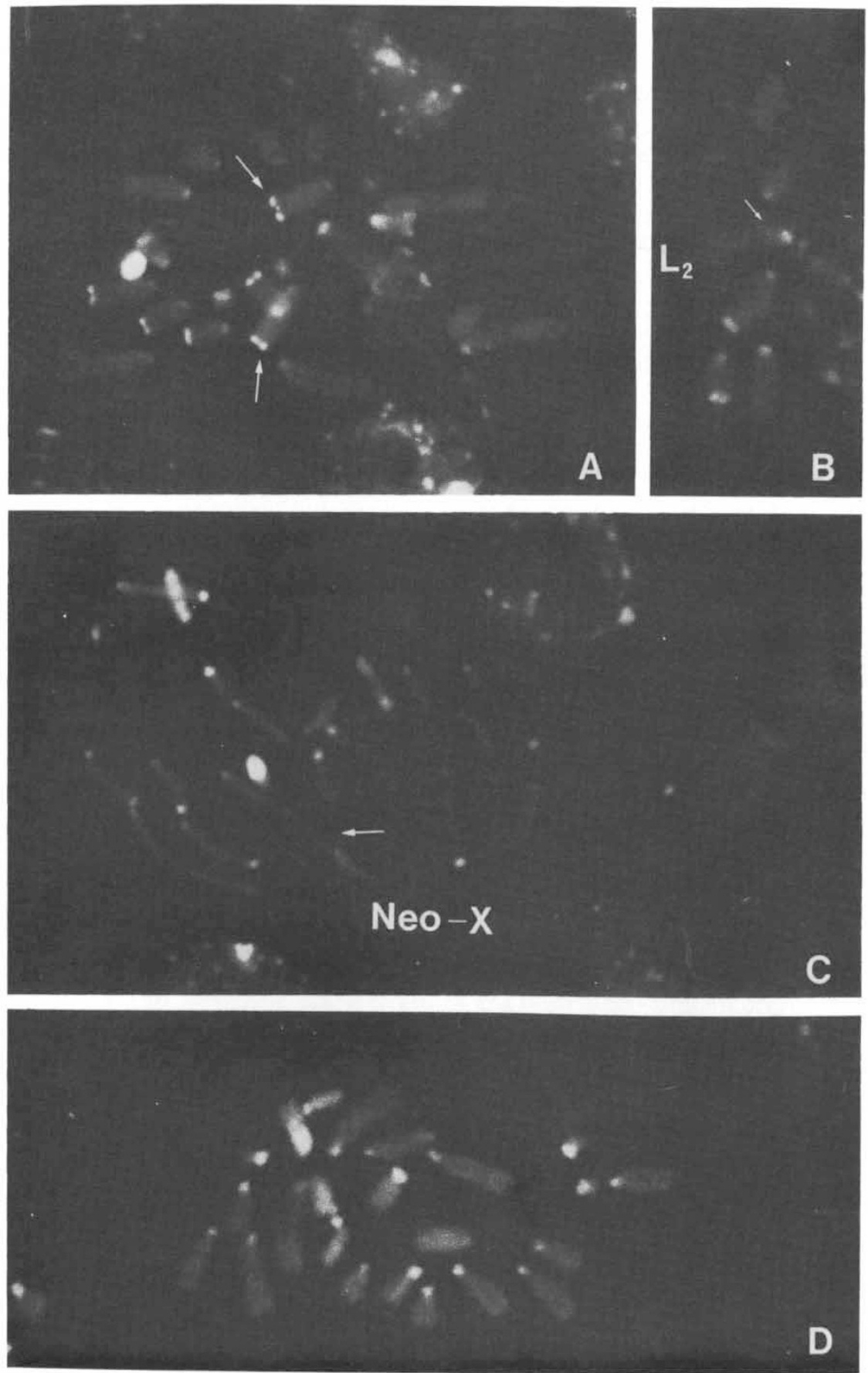

Figure 1 Podisma pedestris after C-banding and Acridine Orange staining. (a) XO race in a female from the Alps. Note the presence of a band in the telomere of both X-chromosomes (arrows) and presence of distal heterochromatin in most of the medium sized pairs. (b) Selected $\mathrm{L}_{2}$ in an individual of the Alps showing an interstitial band which fluoresces in green. This band is not visible when stained with Giemsa. (c) Neo-XY race obtained from an embryo. The distal fluorescent band in the labelled chromosome corresponds to that of the telomere in the X. (d) Podisma pedestris from the Pyrenees. Note large amount of paracentromeric heterochromatin and absence of distal segments in all the chromosomes. 
not as high as that obtained with DA-CMA. Both heterochromatic fluorescent subzones together comprise the heterochromatic region revealed by C-banding plus $\mathrm{AO}$ or Giemsa staining (fig. 1(d); fig. 5).

\section{NOR activity}

The localization of active Nucleolar Organizer Regions (NOR's) with silver staining in Podisma pedestris during meiosis is very difficult, in contrast to other species of grasshoppers, because there is rapid disorganization of the nucleoli early in prophase. Thus, remnants of nucleolus do not usually remain attached to the nucleolar chromosomes. Additionally early diplotenes show very diffuse chromatin which prohibits its precise location.
Fortunately the $\mathrm{X}$-chromosome produces the largest quantity of rRNA and it is possible to distinguish certain differences between the Alpine and the Pyrenean populations. The $\mathrm{XO}$ race from the Alps has a large nucleolus associated with the distal band which shows intense green fluorescence when stained with $\mathrm{AO}$ after $\mathrm{C}$-banding treatment (Fig. 3(d), (c)). The Neo-XY race has two active NOR's, one corresponding to that described above and retained after the fusion and, additionally, a small one detected at the point of the fusion (fig. 3(a), (b), (e)).

By contrast, in the Pyrenean race the production of rRNA in the X-chromosome is located at the centromeric region, associated with the light green fluorescent region visible after $\mathrm{C}$-banding plus AO staining (fig. 3(f), (g)).
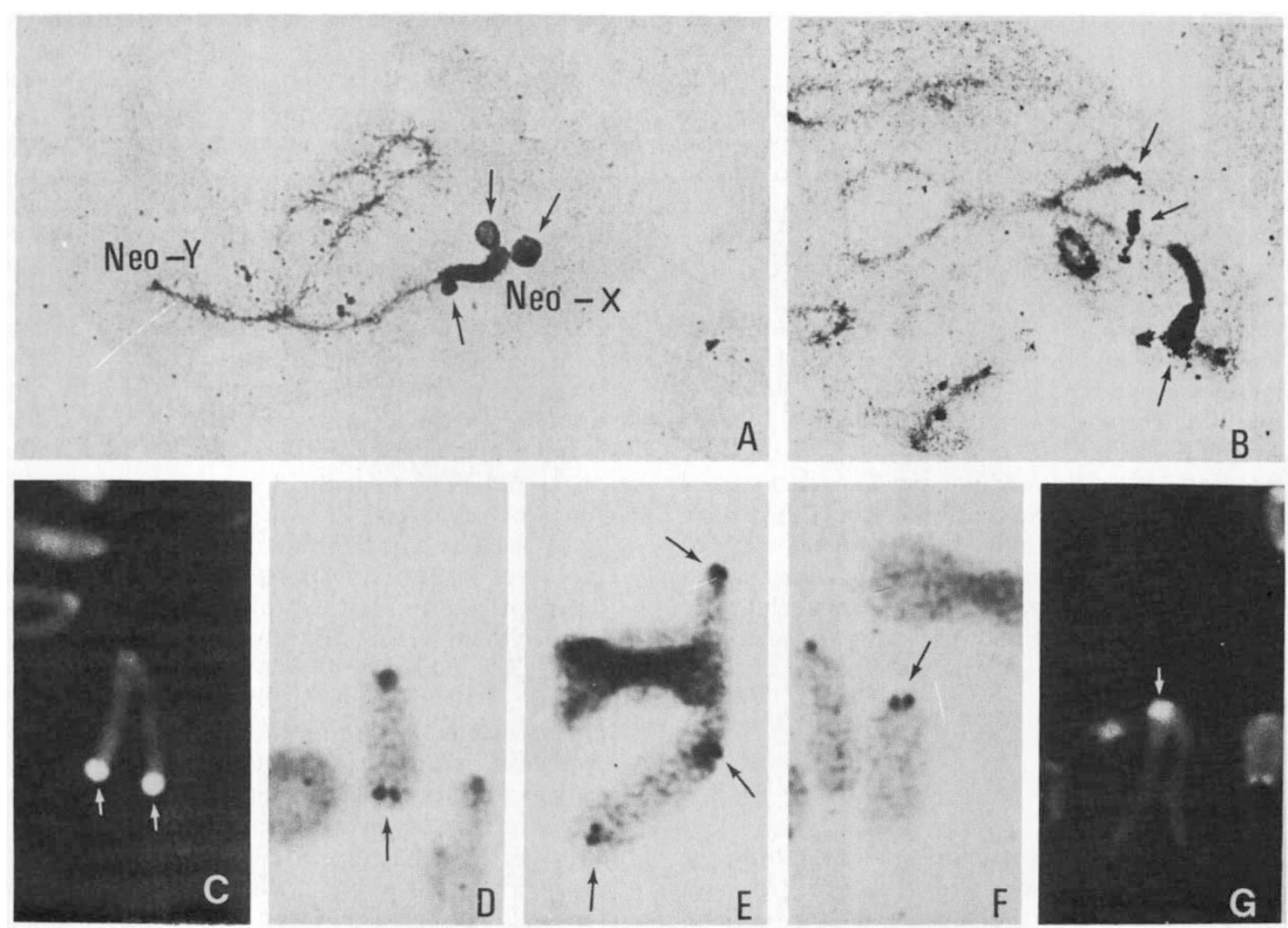

Figure 3 The sex chromosome system of Podisma pedestris. (a), (b) Selected Neo-XY bivalents at diplotene showing large amount of rRNA in the distal regions of the $X$ and small remnants in the region of the fusion but placed on the primitive autosome arms (NeoX and NeoY chromosomes). (c) to (g) Selected sex chromosomes in the different races. (c) C-banding plus AO in XO individuals from the Alps. Distal bands show intense green fluorescence. (d) Selected metaphase 1 chromosome silver stained in the XO form from the Alps. Note NORs in the distal end. (e)The Neo-XY form. NOR activity is maintained at the end of the X. (f) Selected metaphase 1 chromosome silver stained in the XO form from the Pyrenees. Note presence of active NORs in a proximal position. (g) C-banding plus AO in the same race. Note absence of distal band and presence of a fluorescent green band in the proximal region. 
The production of rRNA in these chromosomes is so large, that by increasing silver precipitates after treatment of the chromosomes with $2 \times \mathrm{SSC}$ (Gosálvez et al., 1986), it is possible to find the precise localization of these sites on metaphase-I chromosomes (fig. 3). With this method it is possible to corroborate the results from diplotene stages (compare fig. 3(a), (b) with 3(c)).

Although in both Alps and Pyrenees other autosomes are involved in NOR activity it is difficult to know for certain their number and localization. We know for example that the megameric pair, which is clearly distinguishable by its pycnotic characteristics, is involved with NOR activity in all cases.

\section{The hybrids}

Crosses between Pyrenean and Alpine populations were performed in order to check if the differences in the quantity and quality of heterochromatin and the production of RNAs are associated with any incompatibility in meiosis. Fourth instar males and females from the Pyrenees were crossed in the laboratory with $\mathrm{XO}$ and neo-XY males and females from the Alps. Meiosis of males with hybrid genomes were analyzed. In all cases hybrid males have a standard meiotic process, with complete chromosome pairing in each bivalent. It is possible to recognize the genomes of each parental line in the hybrid. The use of Giemsa C-banding reveals heteromorphism for the different $\mathrm{C}$-heterochromatin content of members of each bivalent (fig. 4(a), (b)). The medium sized bivalents have heterochromatin at the centromeric region in both chromosomes, but only in the distal heterochromatin of the chromosomes from the Alpine populations. The use of fluorescence techniques in neuroblast cells of embryos does not offer any additional information despite the clarity of these chromosomes.

\section{DISCUSSION}

Westerman and Hewitt (1985), using fluorescent counterstaining methods with Hoechst 33258 and CMA demonstrated the relative GC-richness of the $\mathrm{C}$-positive regions at the centromere and distal regions in both $\mathrm{XO}$ and Neo-XY Alpine races of $P$. pedestris. There is also a lack of AT-rich DNA in these genomes. In Pyrenean populations, by contrast, there is no distal heterochromatin in the medium chromosomes, and there is an AT-rich heterochromatic region adjacent to the centric GC in some chromosomes (fig. 5). Additional changes in the $\mathrm{X}$ chromosome differentiate the three chromosome races. A centric fusion between a long autosome and the $\mathrm{X}$ distinguishes a Neo-XY race in the Alps and a change in the position of the active ribosomal cistrons marks the Pyrenean race.

Since the report in 1969 of the centric fusion in some populations from the Alps only one tentative mode of origin of the fusion has been suggested (Westerman and Hewitt, loc. cit.). In this model loss of most of the centric heterochromatin from the original $\mathrm{X}$ chromosome was proposed based on the asymmetrical C-banding pattern of the Neo$X$. Additional support comes from the fact that the ribosomal cistrons placed very close to the centromere of the autosome involved in the fusion are detectable after the rearrangement, indicating the conservatism of the rDNA. The maintenance of all active rDNAs give rise to an unusual trinucleolate bivalent.

The changes suffered by the $\mathrm{X}$ in the Pyrenees seems to be more complex and concern both rearrangements in the heterochromatic material and the position of active NORs. The simplest way to explain this change is by an inversion which moved the active NORs distally placed in the $X$ in the Alpine populations to a centric position in the Pyrenean. The heterochromatin flanking the active X-NORs in Alpine races has been lost in part, given its complete absence in the $\mathrm{X}$ from the Pyrenees and its diminished presence in the centromere. The level of green fluorescence observed in the centromere of the Pyrenean $X$ as that at the distal end of the Alpine $\mathrm{X}$ after $\mathrm{C}$-banding plus AO is not as large.

As well as chromosome rearrangements in the evolution of the species, differences in constitutive heterochromatin may also play an important role in species differentiation (White, 1978; Dover et al., 1982). There are large differences in the amount, distribution and composition of heterochromatic material found in populations of $P$. pedestris from the Alps and Pyrenees. The clear polytypy indicates the existence of a third chromosomal race in the Pyrenees clearly distinguishable from those of the Alps. The presence of this kind of polytypy in other orthopteroids has been reported by several authors, most notably in the Australian genus Warramaba (White et al., 1977); Webb et al., 1978). The differences between Alpine and Pyrenean populations of $P$. pedestris in heterochromatin are rather similar to those in Cryptobothrus chrysophorus. John and King found two distinct chromosomal races from North and South Australia differing in the presence or absence of 

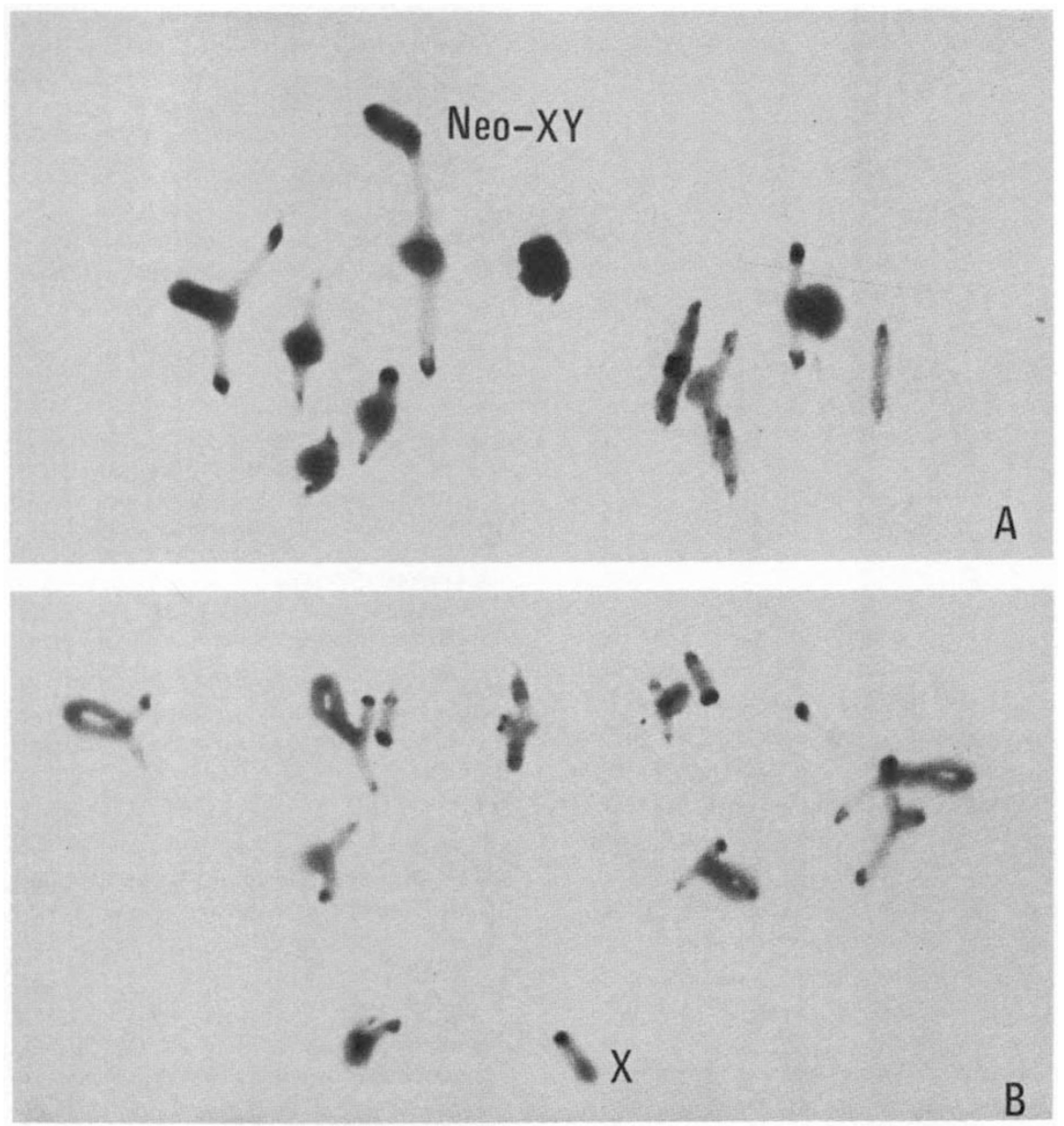

Figure 4 Meiosis of synthetic hybrids in Podisma pedestris. (a) Neo-XY male obtained from a cross between a Neo-XY female from the Alps and an XO male from the Pyrenees. (b) XO male obtained from a cross between an XX female from the Pyrenees and an XO male from the Alps. Note in both cases big differences in the amount of paracentromeric heterochromatin per bivalent. The larger regions are those from the Pyrenean parental line.

heterochromatic blocks on five of the six medium chromosome pairs (John and King, 1977a, b). These races have disjunct geographical distributions and there are no natural hybrids. Synthetic hybrids however have been obtained (John and King, 1980).

On top of such differences, the rearrangements observed in $P$. pedestris have very particular characteristics and could be important in the process of diversification of Podisma in its expansion towards southern areas of Europe. These situations where geographically separated populations show different patterns of genome evolution are indicative of recurrent and cumulative fixation of specific mutations in each isolated area. We need to find a mechanism to explain the existence of AT-plus $\mathrm{GC}$-rich DNA in the paracentromeric regions of some chromosomes from individuals of the Pyrenees and the presence of only GC-rich DNA in populations of the Alps. Clearly both systems have not had the opportunity of co-evolution due to the absence of gene flow between populations. We do not yet know for certain how this enrichment of AT-rich DNA has been originated in the Pyrenean populations. It could correspond to massive duplications of certain gene families such as that reported for Atractomorpha similis where certain repetitive sequences seem to have been divided and dispersed to form massive regions of heterochromatin in different chromosomes (John 

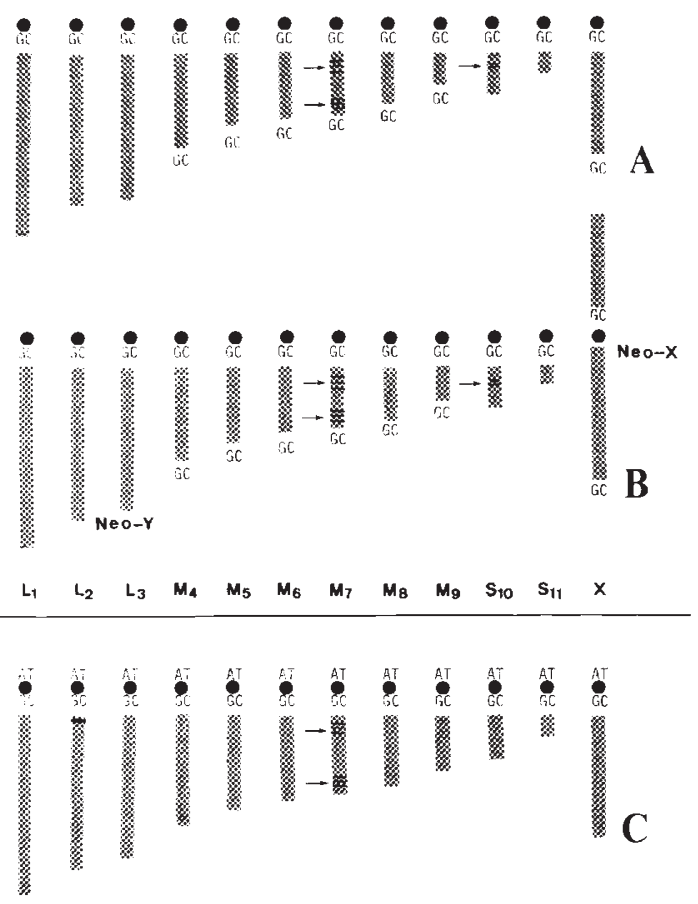

Figure 5 A diagram showing the most important differences in the heterochromatin among the three races of Podisma pedestris. (a) and (b) XO and XY races from the Alps. (c) The Pyrenean race.

et al., 1986). Both gain and loss of heterochromatin could be explained by mechanisms such as saltatory replication or unequal crossing over.

The karyotypic differences noted between Alpine and Pyrenean populations might be expected to interfere with the meiotic behaviour and the viability of hybrids but no such problems were found. However, detailed studies about fitness of hybrids-egg numbers per pod, hatching and nymphal survival-may show a reduction in fitness of hybrids similar to those noted between the XO and Neo-XY in the $P$. pedestris Alpine races.

However the location of active NORs needs to be investigated since the presence of a possible $\mathrm{X}$ inversion might interfere with the meiosis of hybrid females.

Acknowledgements We would particularly like to thank Drs C. López-Fernández, Carlos García de la Vega and Richard Nichols for their help in collecting and hatching the insects, Marise East and Philip Mason for help with rearing, and Marius James for technical assistance. We are grateful to the British Council-Acciones Integradas for a travel grant and to CAICYT (2165/83), NERC and SERC for laboratory support.

\section{REFERENCES}

BARTON, N. AND HEWITT, G. M. 1981 a. A chromosomal cline in the grasshopper Podisma pedestris. Evolution, 35, 10081018.

BARTON, N. AND HEWITT, G. M. $1981 b$. The genetic basis of hybrid variability in the grasshopper Podisma pedestris. Heredity, 47, 367-383.

BELLA, J. L., GARCIA DE LA VEGA, C., LOPEZ-FERNANDEZ, C. AND GOSÁLVEZ, J. 1986. Changes in acridine orange binding and its use in the characterisation of heterochromatic regions. Heredity, 57, 79-83.

DOVER, G. BROWN, S., COEN, E., DALLAS, J., STRACHAN, T. AND TRICK, M. 1982. The dynamics of genome evolution and species differentiation. Genome Evolution, vol. 20. Academic Press, pp. 343-374.

GOSÁlVEZ, J., DE LA TORRE, J., GARC1A DE LA VEGA, C. AND LOPEZ-FERNANDEZ, C. 1986. The effect of double-strength standard saline citrate on silver staining. I. Nucleoli and micronucleoli in the somatic and germ line of the grasshopper Arcyptera fusca. Can. J. Genet. Cytol., 28, 219-226.

HALLIDAY, B., BARTON, N. AND HEWITT, G. M. 1983. Electrophoretical analysis of a chromosomal hybrid zone in the grasshopper Podisma pedestris. Biol. J. Linn. Soc., 19, $51-62$.

HALLIDAY, R. B., WEBB, S. F. AND HEWITT, G. M. 1984. Genetic and chromosomal polymorphism in hybridizing populations of the grasshopper Podisma pedestris. Biol. J. Linn. Soc., 21, 299-305.

HEWITT, G. M. 1975. A sex chromosome hybrid zone in the grasshopper Podisma pedestris (Orthoptera: Acrididae). Heredity, 35, 375-387.

HEWITT, G. M. AND BARTON, N. 1981. The structure and maintenance of hybrid zones as exemplified by Podisma pedestris. Insects Cytogenetics. Symposia of the Royal Entomological Society of London, No. 10. Edited by Blackman, R. L., Hewitt, G. M. and Ashburner, M. Blackwell Scientific Publications, Oxford.

HEWITT, G. M. AND JOHN B., 1972. Interpopulation sex chromosome polymorphism in the grasshopper Podisma pedestris. II. Population parameters. Chromosoma, 37, 2342.

JOHN, B. AND HEWITT, G. M. 1970. Interpopulation sex chromosome polymorphism in the grasshopper Podisma pedestris. I. Fundamental facts. Chromosoma, 31, 291-308.

JOHN, B. AND KING, M. $1977 a$. Heterochromatin variation in Cryptobothrus crysophorus. I. Chromosome differentiatiation in natura! populations. Chromosoma, 64, 219-236.

JOHN, B. AND KING, M. $1977 b$. Heterochromatin variation in Cryptobothrus chrysophorus. II. Patterns of C-banding. Chromosoma, 65, 59-79.

JOHN, B. AND KING, M. 1980. Heterochromatin variation in Cryptobothrus chrysophorus. III. Synthetic hybrids. Chromosoma, 78, 165-186.

JOHN, B., APPELS, R AND CONTRERAS, N. 1986. Population cytogenetics of Atractomorpha similis. II. Molecular characterisation of the distal C-bands polymorphisms. Chromosoma, 94, 45-58.

MORAles-AGACINO, E. 1950. Breves notas sobre los Podismini de la Península Ibérica (Orthoptera: Acrididae). Eos, Tomo extraordinario, 367-384.

NICHOLS, R. A. 1985. Genetical and Ecological differentiation across a hybrid zone. In Gosálvez, J. López Fernández, C. and García de la Vega, C. (eds). Orthoptera, Vol. I, pp. $55-83$.

SCHWEIZER, D. 1976a. DAPI fluorescence of plant chromosomes prestained with Actinomycin D. Exp. Cell Res., 102, 408-412. 
SCHWEIZER, D. 1976 b. Reverse fluorescent chromosome banding with Chromomycin and DAPI. Chromosoma, 58, $307-$ 324.

SCHWEIZER, D. 1980. Fluorescent chromosome bands in plants: applications, mechanisms and implications for chromosome structure. In Davies, D. R. and Hopwood, R. A. (eds). Proc. 4th John Innes Symp. The Plant Genome. pp. 61-72.

SCHWEIZER, D. 1981. Counterstaining-enhanced chromosome banding. Hum. Genet., 57, 1-14.
WEBb, G. C., White, M. J. D., CONTRERAS, N. AND CHENEY, J. 1978. Cytogenetics of the parthenogenetic grasshopper Warramaba (formerly Moraba) virgo and its bisexual relatives. IV. Chromosome banding studies. Chromosoma, 67, 309-339.

WESTERMAN, M. AND HEWITT, G. M. 1985. Chromosome banding in Podisma pedestris. Heredity, 55, 157-161.

WHITE, M. J. D. 1978. Modes of Speciation. W. H. Freeman and Co., San Francisco.

WHITE, M. J. D., CONTRERAS, N. AND WEBB, G. C. 1977. Cytogenetics of the parthenogenetic grasshopper Warramaba virgo (formerly Moraba) and its bisexual relatives. II. Hybridization studies. Chromosoma, 61, 127-148. 